\title{
Tunable Nanoplasmonics
}

\author{
Mohamed A. Swillam $^{1^{*}}$, Rehab Kotb ${ }^{2}$, and Yehea Ismail ${ }^{2}$ \\ ${ }^{1}$ Department of Physics, School of Science and Engineering, \\ The American University in Cairo, Egypt. \\ ${ }^{2}$ Center of Nanoelectronics and Devices (CND) \\ American University in Cairo/ Zewail City of Science and Technology \\ Cairo, Egypt \\ *corresponding author, E-mail: m.swillam@aucegypt.edu
}

\begin{abstract}
In this paper, we present novel mechanisms for tuning and controlling the response of novel plasmonic filter using metal-insulator-metal (MIM) configuration. These mechanisms allow for full control on the transmission response from these waveguide based structures. This control can be done electrically or mechanically. The applications and advantages of these novel schemes are discussed in details. Wideband tuning range has been obtained using these schemes.
\end{abstract}

\section{Introduction}

Plasmonics waveguides have shown unique advantage to guide the light while confined in subwavelength area. This unique advantage makes the plasmonics waveguide attractive candidate for various applications. These applications include high density integration for optical interconnects, on-chip biosensing, and nonlinear applications.

Among various plasmonics waveguides, metal-insulator metal (MIM) is considered as a suitable candidates for the aforementioned applications. The 3D version of this configuration is the plasmonic slot waveguide (PSW) as shown in Fig.1.

This waveguide configuration has been utilized in various proposed configurations to attain different filter responses. However practical realization of these waveguides is challenging. Moreover, good coupling efficiency to this waveguide with few nanometers gap is the bottleneck of using such waveguides. Recently, a novel coupling mechanism is proposed, fabricated and tested with more than $70 \%$ coupling efficiency [1], [2].

This novel coupling mechanism provides a suitable solution for accessing and fabricating these waveguides and integrates it with silicon waveguides on the same substrate. This opens the door for various applications that can be obtained using a mesh or interleaved networks of these waveguides. These networks have been recently utilized to obtain various functionalities [3], [4]. Most of these functionalities are, however, passive applications. In general tuning or modulating the response of MIM based structures are challenging.

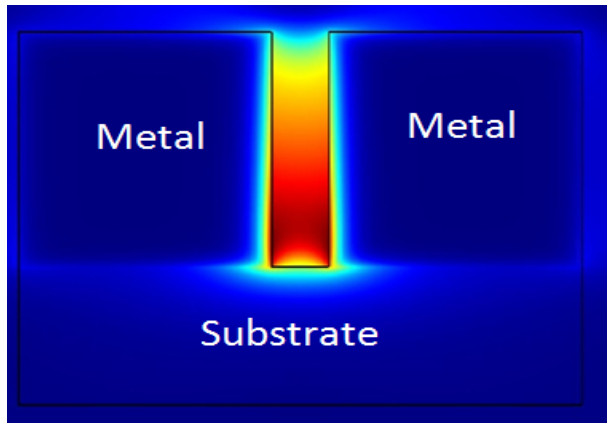

Fig.1. The field profile in a $50 \mathrm{~nm}$ slot mode waveguide with an air gap between two metal layers

Optical tunable filters have a wide range of applications in sensing and communication systems. The advantages of having wide tunable range can be used in tunable laser, channel monitoring, optical switching, and wavelength division multiplexing/ demultiplexing. Quick and accurate tunability is also required in many biomedical diagnoses, such as optical coherence tomography (OCT) [5]. In general, tunable filters can be used in Biochemical Analysis of Human's Fluids [6]. Furthermore, tunable filter can be used in monitoring and administrating communication channels. Increasing the capacity of optical networks can be achieved using wavelength division Multiplexing (WDM). Using WDM, high bit rate and large number of communication channels can be accomplished [7]. Narrow band, wide tuning range optical filters are key components in WDM. Tunable filters manage us to use single small device to be able to switch between different channels in the demultiplexres [8].

In addition, due to the high capacity transmission of optical communication systems, the optical switches are considered as good solution of the electronic switches speed limitation [9]. Optical switches main advantage is its ability 
to route the signals without the need of electronic conversion which leads to independence on data rate and protocols. By this way, the switching speed is increased which results in increasing network throughput, and the needed network equipment are decreased leading to decreasing the overall operating power. Easy, accurate, and fast tuning mechanisms are required for aforementioned applications.

Even though plasmonic devices provide excellent confinement and high density integration, obtaining the aforementioned applications using plasmonics devices are highly challenging. For example plasmonics switching mechanism has been proposed using electro optic effect as shown in [13],[14]. These designs are based on ring resonator in which the output response depends mainly on the coupling between the ring and adjacent waveguide. The range of tunabilty is very small. The fabrication process of narrow coupling gaps between the ring and the waveguide is very critical and may lead to significant size variation. Other complicated plasmonic switching technique is proposed in [16] which based on Molecular Machine-Au Nanodisk. Besides, some other switching techniques based on MachZehnder interferometer [17], and five-layer plasmonic waveguide [18] are proposed. However, these devices lack the ability of movement and mechanical tuning.

The ability to provide tunable filter using plasmonics is however more changeling than optical switching especially if wide tunable range is required. Few methodologies for tunable plasmonics circuits have been proposed. As reported in [19], thermal tuning can be used in sub-wavelength bandstop plasmonic filters. In addition, tuning mechanism using liquid crystal waveguide is proposed in [20]. Other filtering techniques are proposed in [21],[22]; however, these techniques lack the ability of tuning in easy and accurate manner

In this paper we propose a novel device that can be easily tuned using a simple mechanism to control and tune its response. This structure lends itself to different tunable approaches. A possible tuning mechanism is through micro/Nano electromechanical systems (MEMS/NEMS). In this mechanism an inline resonance filter is utilized with tunable dimensions. Varying the length of the resonator is done through mechanical motion. A wide tunable range can be obtained by changing the resonator dimensions. Another approach for tuning can be done through electro-optic effect by changing the refractive index inside the slot.

These approaches for tunability open the door for wide range of applications such as tunable filters, tunable lasers, spectrum analyzer, and biosensing.

\section{MIM Optical Resonator}

A simple design of an inline optical Fabry-Perot resonator is shown in Fig. 2. The transverse metal sides' acts like mirrors or reflectors for the resonator. Controlling the gap width $W_{1}$ and $W_{2}$ allows for controlling the value of the reflectivity of the mirror. The output resonance response of the filter is controlled by the dimension of the resonance. The resonance condition of the filter is given by:

$$
m \lambda_{r}=2 n_{\text {eff }} L
$$

where $n_{\text {eff }}$ is the effective index inside the resonator, $\lambda_{r}$ is the resonance peak wavelength and $L$ is the resonator length.

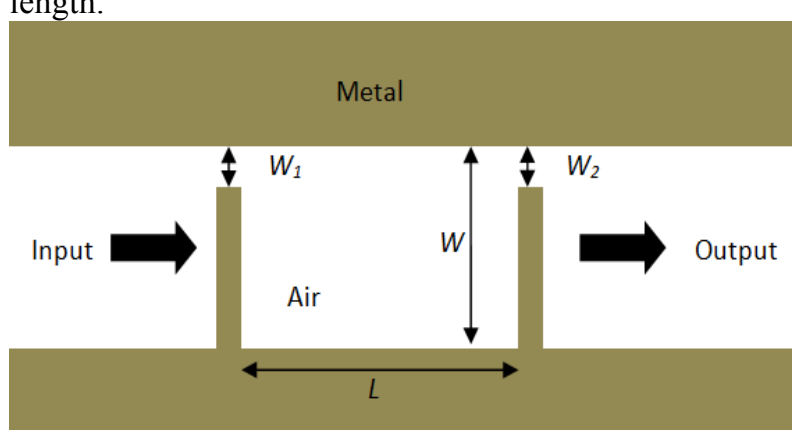

Fig.2. Schematic of the tunable plasmonic filter

Thus, the resonance condition depends on the length of the resonator $L$ and the effective index $n_{\text {eff. Controlling }}$ these parameters allow for full control on the resonance wavelength and provide full tuning ability of the structure.

\subsection{Tuning Mechanism}

Tuning the wavelength is required to satisfy a broad range of filtering applications to get the desired signal. A reconfigurable highly selective filter is considered as a main challenge in many optical applications. Easy, simple, and high effective tuning methods are discussed in the next few lines to provide a simple mechanism to get the desired wavelength. From equation (1), we can see that the resonance wavelength can be controlled by changing the cavity length or the effective index. The cavity length can be changed mechanically. However, the effective index can be changed either mechanically or electro-optically.

\subsubsection{Mechanical Tuning}

\subsubsection{Controlling the cavity length}

Tunability can be achieved by changing the optical path length; then, the round trip of the optical cavity can be controlled. By tuning the cavity length, we can manage the wavelength of the output signal. Controlling the resonator length can be achieved by moving the transverse metal sides of the filter shown in Fig.2. MEMS/NEMS can be used to move these metal sides forward and backward to get the desired response. The narrow bandwidth of the output signal gives us the ability to fine tune this filter to have the output peak power at adjacent wavelengths with high accuracy. In addition, the ease of changing the output wavelength provides wide range of tunability. By changing the length of the filter (L) from $0.5 \mu \mathrm{m}$ to $1 \mu \mathrm{m}$, we can get the resonance peak at different wavelengths as shown in Fig. 3. 


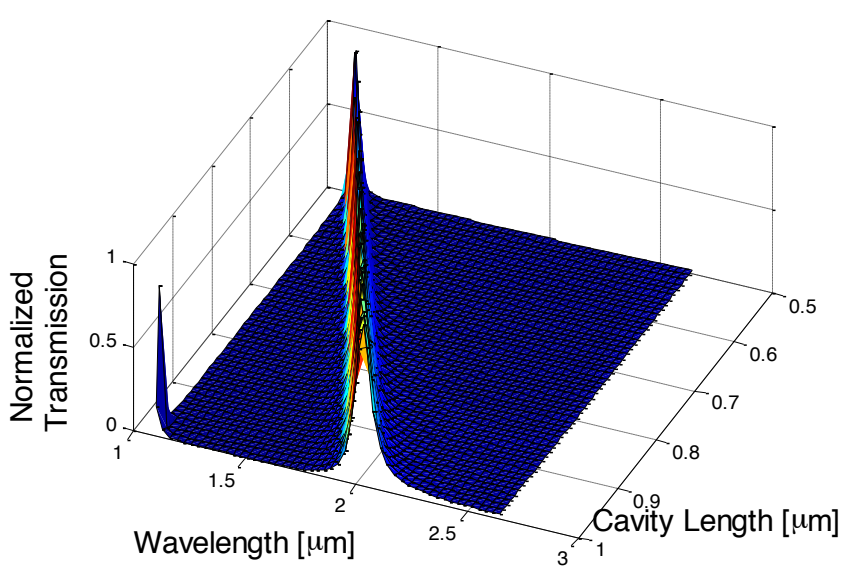

Fig.3. Tuning the length of the resonator controlling the resonance wavelength

\subsubsection{Controlling the cavity width}

In addition to changing the optical path length by changing the cavity length, we can change it by controlling the effective index inside the resonator cavity. As we stated before, the effective index can be controlled mechanically or electro-optically. Changing the effective index mechanically can be done by changing the cavity width. Moving a rectangular metal inside the filter cavity upward and downward is able to control the effective index of the dielectric core and hence, the output wavelength. By changing the width of the cavity (W) from $50 \mathrm{~nm}$ to $250 \mathrm{~nm}$, we can get the resonance peak at different wavelengths as shown in Fig. 4.

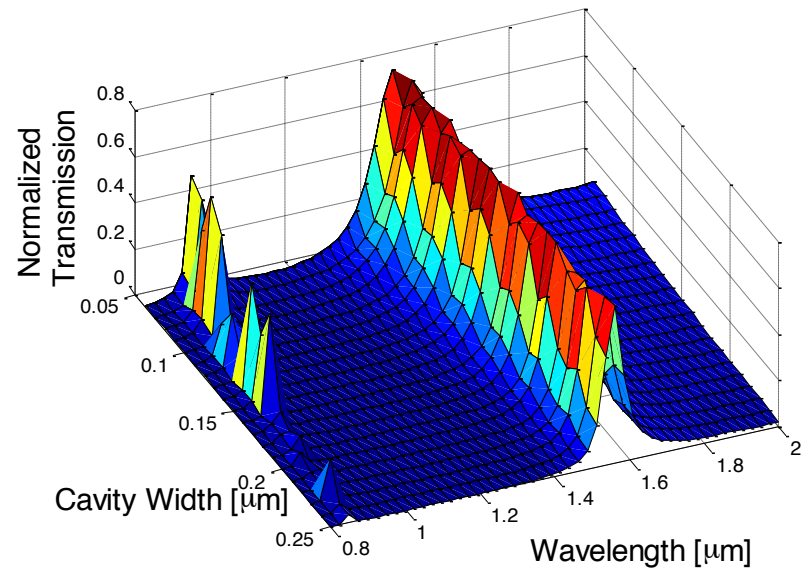

Fig.4. Tuning the width of the resonator controlling the resonance wavelength

\subsubsection{Electrical Tuning}

In addition to controlling the effective index mechanically, we can control it electrically by applying an electric field to the dielectric core [10],[11]. By applying a voltage difference between the two metal plates of the filter, the value of the applied electric field can be changed and consequently the effective index. Electrically injecting electrons and holes in the dielectric core using embedded $\mathrm{p}-\mathrm{i}-\mathrm{n}$ junction is an effective way to change the core effective index [10]. By changing the effective index from 1 to 2, resonance peak can be tuned as shown in Fig.5.

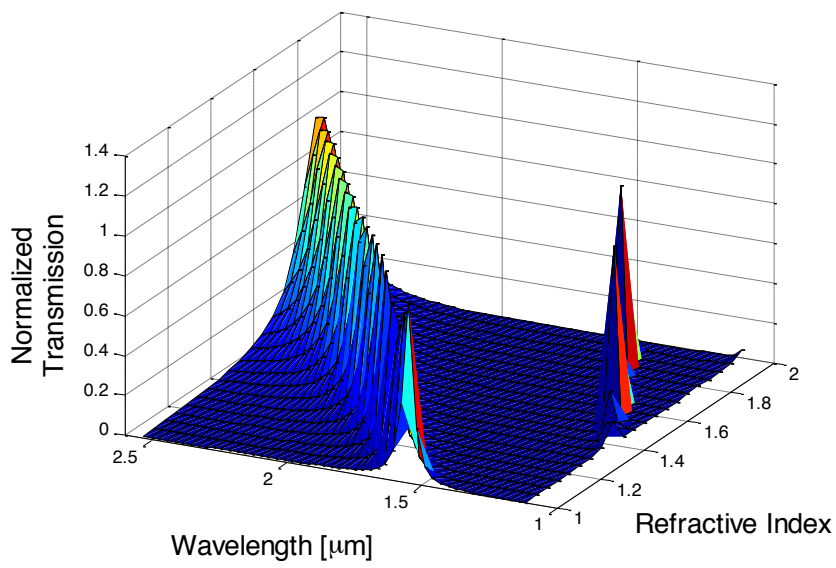

Fig. 5.The results of tuning the resonance wavelength electrically

Moreover, this filter can be used in biomedical sensing. By changing the dielectric material inside the filter, the index will change resulting in changing the resonance wavelength.

\section{Discussion}

Many applications have been empowered from the properties of surface plasmon polarities waveguides due to the ability to confine the light at subwavelength scale. On chip interconnects, biomedical sensors, wavelength multiplexers/ demultiplexers, optical switches, and a lot of other photonic applications are wavelength dependent and need kind of wavelength filtering. For the purpose of minimization, integration is needed with high efficiency and good accuracy. Tunable filter can be the appropriate solution for wavelength selectivity. In this paper, we proposed a design of a nano-tunable filter that provides wide range of wavelength selectivity using simple mechanisms. This filter consists of dielectric core sandwiched between two metal layers. By changing the filter dimensions or the dielectric effective index, the resonance wavelength can be controlled. Mechanically, we can control the length of the filter by adjusting the position of the movable plate (1) as shown in Fig.6.

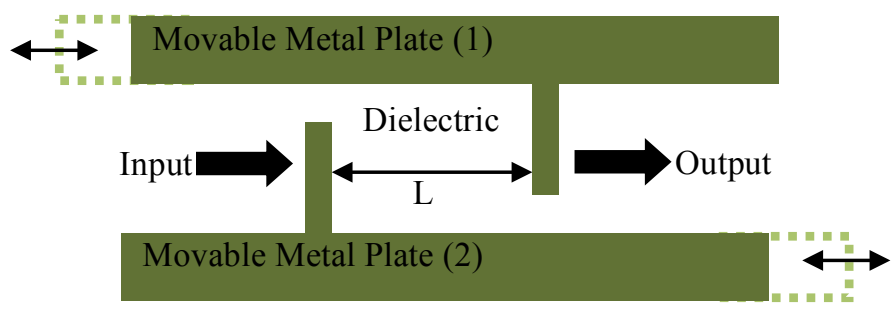

Fig. 6. Schematic diagram to the horizontal movement of the plasmonic filter

Furthermore, we can move both metal plates (1) and (2) relative to each other in order to get a broad range of 
selected resonance wavelengths. Adjusting the length has a direct impact on the filer output as illustrated before in Fig.3. From Fig.7, we can see the proportional relation between

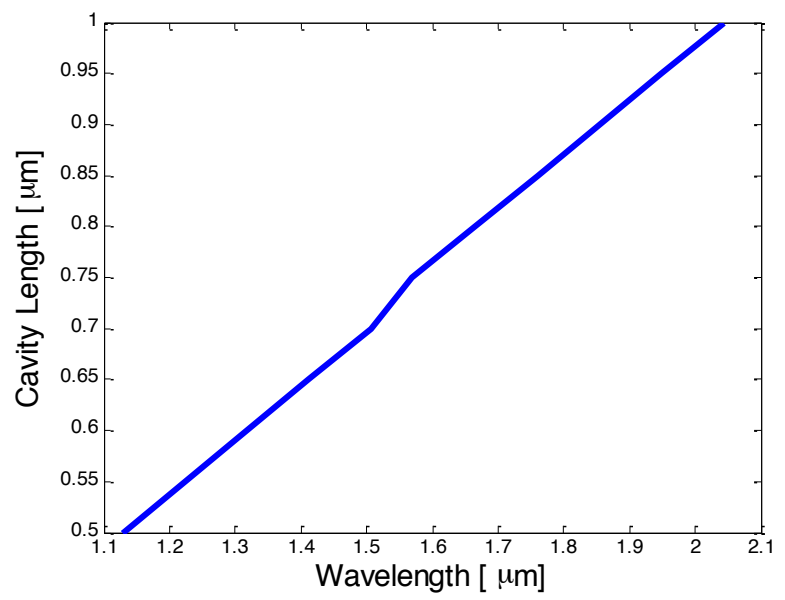

the resonance wavelength and the cavity length.

Fig. 7. The relation between the Wavelength and the Cavity length

Besides the horizontal movement, we can add a vertical movement to change the filter width as shown in Fig.8. As a consequence, the effective index of the dielectric core of the filter will change resulting in changing the position of the output peak as discussed in Fig.4. In addition to the mechanical controlling, the electro-optical controlling can be used in changing the effective index of the dielectric core. As we can see in Fig.9, by changing the applied electric volt, the effective index can be tuned easily and accurately.

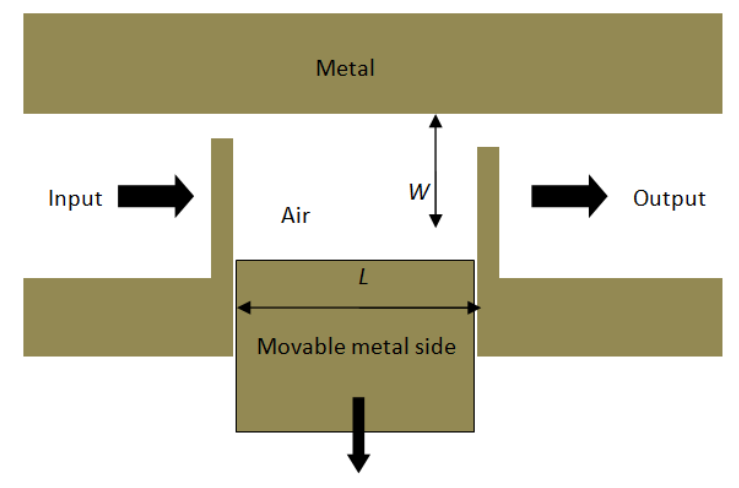

Fig.8. Schematic diagram to the vertical movement of the plasmonic filter

There are different methods to adjust the effective index using electric field, for example Pockels effect, and Kerr effect. In the Pockels effect method, the refractive index is linearly proportional to the strength of the applied electric field. In Kerr effect method, the refractive index is proportional to the square of the applied electric field [12]. The aforementioned simple and easy mechanisms to control the resonance frequency of the filter open the door for many plasmonic applications.

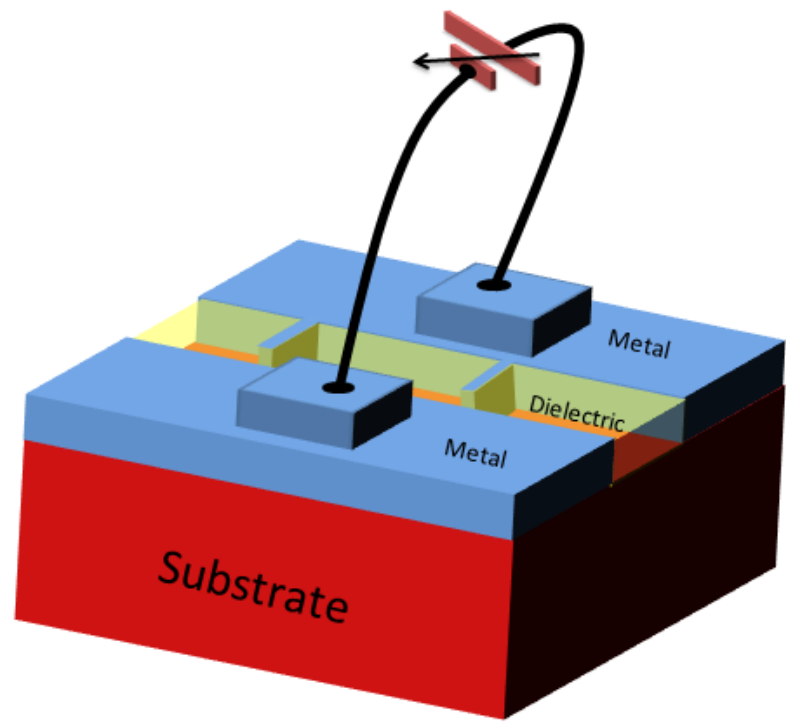

Fig.9. Schematic diagram to changing the electric field in the cavity of the plasmonic filter

\section{Conclusions}

A novel tunable filter design to control the transmission response is proposed. Tuning the resonance wavelength can be achieved mechanically by changing the cavity length or the cavity width, or electro optically by applying an electric field to the dielectric inside the filter. This ability to tune the resonance wavelength is very attractive to many optical applications.

\section{References}

[1] B. Lau, M. A. Swillam, and A. S. Helmy, "Hybrid orthogonal junctions: wideband plasmonic slot - silicon wire couplers," Optics Express, vol. 18, no. 26 , pp. 27048-27059, Dec. 2010.

[2] Charles Lin, Herman M. K. Wong, Benedict Lau, Mohamed A. Swillam, and Amr S. Helmy, "Efficient broadband energy transfer via momentum matching at hybrid junctions of guided-waves" Appl. Phys. Lett. 101, 123115 (2012).

[3] M. A. Swillam, and A. S. Helmy, "Feedback effect in plasmonic slot waveguides examined usnng closed form," Photonics Technology lett., vol.19,no. 24, pp 497-499, March 2012.

[4] C. Lin, M. A. Swillam, and A. S. Helmy, "Analytical model for metal-insulator-metal mesh waveguide architectures," J. Opt. Soc. Am. B, vol. 29, no. 11, 3157-3169 (2012).

[5] Jingjing Sun and Huikai Xie, "MEMS-Based Endoscopic Optical Coherence Tomography," International Journal of Optics, vol. 2011, Article ID 825629, 12 pages, 2011.

[6] Pinheiro, C.; Rocha, J. G.; Goncalves, L.M.; Lanceros-Mendez, S.; Minas, G., "A Tunable Fabry-Perot Optical Filter for Application in Biochemical Analysis of Human's Fluids," Industrial Electronics, IEEE International Symposium on, vol.4, no., pp.2778,2783, July 2006.

[7] Hailiang Zhang, Yan Betremieux, John Noto, Robert Kerr, "Novel tunable liquid crystal Fabry-Perot filters for Fiber-Optical system," 
Proc. of SPIE, Vol. 4583,p. 64-72, Metro and Access Networks, 2001.

[8] Tran, A.T.T.D.; Lo, Y.H.; Zhu, Z.H., "Surface-micromachined FabryPerot tunable filter for wavelength-division multiplexing systems," Lasers and Electro-Optics, vol., no., pp.346,June 1996.

[9] Papadimitriou, Georgios I.; Papazoglou, C.; Pomportsis, Andreas S., "Optical switching: switch fabrics, techniques, and architectures," Lightwave Technology, vol.21, no.2, pp.384,405, Feb. 2003.

[10] Qianfan Xu, Bradley Schmidt, Sameer Pradhan \& Michal Lipson, "Micrometre-scale silicon electro-optic modulator," Nature 435, 325-327 (2005).

[11] Zhao, Qibin; Haines, Andrew; Snoswell, David; Keplinger, Christoph; Kaltseis, Rainer; Bauer, Siegfried; Graz, I.; Denk, Richard; Spahn, Peter; Hellmann, Goetz; Baumberg, Jeremy J., "Electric-field-tuned color in photonic crystal elastomers," Applied Physics Letters, vol.100, no.10, pp.101902,101902-4, Mar 2012

[12] http://www.rp-photonics.com

[13] Krasavin, A.V.; Zayats, A.V., "Electro-optic switching element for dielectric-loaded surface plasmon polariton waveguides," Applied Physics Letters, vol.97, no.4, pp.041107,041107-3, Jul 2010

[14] Cetin, A.E.; Yanik, Ahmet A.; Mertiri, Alket; Erramilli, Shyamsunder; Mustecaplioglu, Ozgur E.; Altug, Hatice, "Field-effect active plasmonics for ultracompact electro-optic switching," Applied Physics Letters, vol.101, no.12, pp.121113,121113-4, Sep 2012

[15] S. Randhawa, S. Lachèze, J. Renger, A. Bouhelier, R. E. de Lamaestre, A. Dereux, and R. Quidant, "Performance of electro-optical plasmonic ring resonators at telecom wavelengths," Opt. Express20(3), 2354-2362 (2012).

[16] Yue Bing Zheng; Ying-Wei Yang; Jensen, L.; Lei Fang; Juluri, B.K.; Flood, A.H.; Weiss, P.S.; Stoddart, J.F.; Huang, Po-Hsun, "A plasmonic switch based on molecular machine$\mathrm{Au}$ nanodisk complexes," Lasers and Electro-Optics, 2009 and 2009 Conference on Quantum electronics and Laser Science Conference. CLEO/QELS 2009. Conference on, vol., no., pp.1,2, 2-4 June 2009

[17] S. Papaioannou, D. Kalavrouziotis, K. Vyrsokinos, J.-C. Weeber, K. Hassan, L. Markey, A. Dereux, A. Kumar, S. I. Bozhevolnyi, M. Baus, T. Tekin, D. Apostolopoulos, H. Avramopoulos, and N. Pleros, "Active plasmonics in WDM traffic switching applications," Sci Rep2, 652 (2012).

[18] Ozaki, J.; Murata, H.; Takahara, J.; Okamura, Y., "New electro-optic switch using symmetric five-layer plasmonic waveguide with light-coupling slot-antennas," Lasers and Electro-Optics (CLEO), 2012 Conference on, vol., no., pp.1,2, 6-11 May 2012

[19] Tao, Jin; Wang, Qi Jie; Hu, Bin; He, Xiao Yong; Zhang, Ying, "Tunable subwavelength terahertz plasmonic stub waveguide filters," Nanoelectronics Conference (INEC), 2013 IEEE 5th International, vol., no., pp.455,458, 2-4 Jan. 2013

[20] D.C. Zografopoulos, R. Beccherelli, A.C. Tasolamprou, E.E. Kriezis, "Liquid-crystal tunable waveguides for integrated plasmonic components," Photonics and Nanostructures Fundamentals and Applications, Volume 11, Issue 1, February 2013

[21] Lu, H., Liu, X., Mao, D., Wang, L. \& Gong, Y. “Tunable band-pass plasmonic waveguide filters with nanodisk resonators". Optics Express 18, 17922-17927 (2010).

[22] J. Tao, X. G. Huang, X. Lin, Q. Zhang, and X. Jin, "A narrowband subwavelength plasmonic waveguide filter with asymmetrical multiple-teeth-shaped structure," Opt. Express 17(16), 13989-13994 (2009) 